\title{
Erratum to: Higher-order optimality conditions for weakly efficient solutions in nonconvex set-valued optimization
}

\author{
Q. L. Wang · S. J. Li • K. L. Teo
}

Published online: 22 October 2011

(C) Springer-Verlag 2011

\section{Erratum to: Optim Lett (2010) 4:425-437 \\ DOI 10.1007/s11590-009-0170-5}

Unfortunately, the incorrect version of [1, Theorem 4.3] was published. The correct version of [1, Theorem 4.3] is given in this paper.

By employing the generalized higher-order contingent derivatives of set-valued maps, Wang et al. [1] established a sufficient optimality condition of weakly efficient solutions for $(S V P)$ :

$$
(S V P) \begin{cases}\min & F(x), \\ \text { s.t. } & G(x) \bigcap(-D) \neq \emptyset, x \in E .\end{cases}
$$

Theorem 1 (see [1, Theorem 4.3]) Assume that the following conditions are satisfied:

(i) $\left(u_{i}, v_{i}, w_{i}\right) \in\left\{0_{X}\right\} \times C \times D, i=1,2, \ldots, m-1$;

(ii) There exists $(\Gamma, L) \subset\left(C^{+} \times D^{+}\right) \backslash\left(0_{Y^{*}}, 0_{Z^{*}}\right)$ such that

The online version of the original article can be found under doi:10.1007/s11590-009-0170-5.

Q. L. Wang $(\varangle) \cdot$ S. J. Li

College of Mathematics and Statistics, Chongqing University, 400044 Chongqing, China

e-mail: Wangq197@126.com

S. J. Li

e-mail: lisj@cqu.edu.cn

Q. L. Wang

College of Sciences, Chongqing Jiaotong University, 400074 Chongqing, China

K. L. Teo

Department of Mathematics and Statistics, Curtin University of Technology,

G.P.O. Box U1987, Perth, WA 6845, Australia

e-mail: K.L.Teo@curtin.edu.au 
$C:=\{x \in Y \mid f(x) \geq 0$, for any $f \in \Gamma\}, D:=\{x \in Z \mid g(x) \geq 0$, for any $g \in L\}$,

$$
\sup _{(f, g) \in(\Gamma, L)}\left\{\frac{f\left(0_{Y}\right)+g\left(-z_{0}\right)}{f(e)+g(k)}\right\}=0,
$$

and

$$
\sup _{(f, g) \in(\Gamma, L))}\left\{\frac{f(y)+g(z)}{f(e)+g(k)}\right\}>0
$$

forany $(y, z) \in G-D^{(m)}\left(F_{+}, G_{+}\right)\left(x_{0}, y_{0}, z_{0}, u_{1}, v_{1}, w_{1}, \ldots, u_{m-1}, v_{m-1}, w_{m-1}\right)(x)$, $x \in \operatorname{dom}\left[G-D^{(m)}\left(F_{+}, G_{+}\right)\left(x_{0}, y_{0}, z_{0}, u_{1}, v_{1}, w_{1}, \ldots, u_{m-1}, v_{m-1}, w_{m-1}\right)\right]$.

Then $\left(x_{0}, y_{0}\right)$ is a weakly efficient solution of $(S V P)$.

We would like to explain the mistake in [1, Theorem 4.3] and correct it.

On the one hand, it follows from [1, Proposition 3.2] that $0_{X} \in \operatorname{dom}\left[G-D^{(m)}\left(F_{+}\right.\right.$, $\left.\left.G_{+}\right)\left(x_{0}, y_{0}, z_{0}, u_{1}, v_{1}, w_{1}, \ldots, u_{m-1}, v_{m-1}, w_{m-1}\right)\right]$ and $\left(0_{Y}, 0_{Z}\right) \in G-D^{(m)}\left(F_{+}\right.$, $\left.G_{+}\right)\left(x_{0}, y_{0}, z_{0}, u_{1}, v_{1}, w_{1}, \ldots, u_{m-1}, v_{m-1}, w_{m-1}\right)\left(0_{X}\right)$. Therefore, for any $\Gamma \subset C^{+}$ and $L \subset D^{+}$with $\Gamma \times L \neq\left\{0_{Y^{*}}\right\} \times\left\{0_{Z^{*}}\right\}$, the condition (2) in Theorem 1 never holds.

On the other hand, the condition (1) can be simply written as

$$
z_{0}=0_{Z}
$$

Indeed, $(3) \Rightarrow(1)$ is obvious. In what concerns the implication $(1) \Rightarrow(3)$, it follows from $z_{0} \in-D$ that $g\left(-z_{0}\right) \geq 0$, for all $g \in L \subset D^{+}$. Thus, if (1) holds, then for all $(f, g) \in \Gamma \times L$, we have

$$
0 \leq \frac{f\left(0_{Y}\right)+g\left(-z_{0}\right)}{f(e)+g(k)} \leq \sup _{\left(f^{\prime}, g^{\prime}\right) \in \Gamma \times L}\left\{\frac{f^{\prime}(y)+g^{\prime}(z)}{f^{\prime}(e)+g^{\prime}(k)}\right\}=0
$$

which implies $g\left(-z_{0}\right)=0$, for all $g \in L$. This means that $-z_{0}, z_{0} \in\{x \in Z \mid g(x) \geq$ $0, \forall g \in L\}=D$. Since $D$ is pointed, we conclude that $z_{0}=0_{Z}$.

Thus the corrections of [1, Theorem 4.3] are as follows.

Theorem 2 Assume that the following conditions are satisfied:

(i) $\left(u_{i}, v_{i}, w_{i}\right) \in\left\{0_{X}\right\} \times C \times D, i=1,2, \ldots, m-1$;

(ii) $z_{0}=0_{Z}$ and there exist $\Gamma \subset C^{+}$and $L \subset D^{+}$with $\Gamma \times L \neq\left\{0_{Y^{*}}\right\} \times\left\{0_{Z^{*}}\right\}$ such that

$$
C:=\{x \in Y \mid f(x) \geq 0, \text { for any } f \in \Gamma\}, \quad D:=\{x \in Z \mid g(x) \geq 0, \text { for any } g \in L\}
$$

and

$$
\sup _{(f, g) \in \Gamma \times L}\left\{\frac{f(y)+g(z)}{f(e)+g(k)}\right\}>0
$$


for any $(y, z) \in G-D^{(m)}\left(F_{+}, G_{+}\right)\left(x_{0}, y_{0}, z_{0}, u_{1}, v_{1}, w_{1}, \ldots, u_{m-1}, v_{m-1}, w_{m-1}\right)(x)$ with $(y, z) \neq\left(0_{Y}, 0_{Z}\right), x \in \operatorname{dom}\left[G-D^{(m)}\left(F_{+}, G_{+}\right)\left(x_{0}, y_{0}, z_{0}, u_{1}, v_{1}, w_{1}, \ldots, u_{m-1}\right.\right.$, $\left.\left.v_{m-1}, w_{m-1}\right)\right]$.

Then $\left(x_{0}, y_{0}\right)$ is a weakly efficient solution of (SVP).

Proof The proof follows on the lines of [1, Theorem 4.3].

Acknowledgments This research was partially supported by the National Natural Science Foundation of China (No. 10871216 and No.11071267), Chongqing Natural Science Foundation Project of CQ CSTC(cstcjjA00019) and Science and Technology Research Project of Chong Qing Municipal Education Commission (KJ100419). The authors would like to thank anonymous referees for their valuable comments and suggestions, which helped to improve the paper.

\section{Reference}

1. Wang, Q.L., Li, S.J., Teo, K.L.: Higher-order optimality conditions for weakly efficient solutions in nonconvex set-valued optimization. Optim. Lett. 4, 425-437 (2010) 